\title{
Woman Volleyball Players Control Body Weight's Dietary Nutrition, Body Composition and Blood Biochemical Index Analysis
}

\author{
Xiaolin Wang ${ }^{*}$
}

School of Physical Education, Hubei Engineering University, Xiaogan, Hubei 432000, China

\begin{abstract}
Volley ballis a sub-weight-level sport, and weight requirements of the project are in the pre-race period of time to reduce body weight. Option volleyball team for dietary analysis of the survey was to understand Athletes in weight control by pre-and post-meal nutrition through body composition and blood biochemical indicators to understand the health status of athletes. The unreasonable parts of dietary of the recommendations put forward guidance, the analysis of the athletes from the normal diet to control weight of the period of dietary changes in body composition for athletes and blood biochemical parameters, body composition and blood biochemical changes can be reflected in athletes The nutritional meals, and can change their diet to improve the situation for athletes to maintain good athletic ability and competitive level to ensure that material. This topic took 16 players in the women's volleyball team for the study in Sports University s, including 10 master athletes, six-level athletes. This study used a three-day dietary survey weighed law, the use of the State Sports General Administration of Sports Medicine Institute for the development of the dietary data analysis software to deal with meals, the Beijing Sports University research center's Inbody 3.0 body composition is tested athletes from Xiyuan Hospital blood biochemical markers to detect blood biochemical indicators, the final on access SPSS 13.0 statistical data processing.
\end{abstract}

Keywords: Biochemical indicators, body composition, control weight, dietary nutrition, volleyball.

\section{INTRODUCTION}

In order to better service for sports training, it is necessary to carry out the dietary nutrition survey and blood biochemical index analysis of women's volley ball team. The dietary nutrition survey analysis methods are diverse; all sorts of dietary nutrition survey methods are used in different degree during practice. Home and abroad the three days of dietary weighing method is used for nutrition survey, the accuracy of this method is higher, and the operation of it is relatively simple. In view of the little survey analysis on volleyball players' dietary nutrition, it's especially less in the normal diet and weight control period of dietary survey analysis. The nutrition of normal diet to weight reduction period diets of athletes can affect body composition and blood biochemical indicators to change, and the change of body composition and blood biochemical indexes can reflect the dietary nutrition of the athletes, and reasonable improvement of the dietary nutrition can be taken in case of it, so as to provide material guarantee to keep good athletic ability and competitive level for athletes.

Choosing the dietary nutrition survey of volleyball team for analysis is to understand the current dietary nutrition status of athletes, and understand the health of athletes through body composition and blood biochemical index.
Instructive proposals are put forward for unreasonable places in the dietary nutrition, at the same time making reasonable dietary nutrition for the control weight reduction is provided reference basis. Analysis the normal athletes diet and weight control stage change characteristics of body composition and blood biochemical indexes, fumble through dietary adjustment to reduce body fat and increase the methods of lean body mass ratio. Using body composition and blood biochemical indicators to auxiliary analyze the rationality of the dietary nutrition, and the dietary lipid control suggestions are put forward.

\section{MATERIAL AND METHODS}

\subsection{The Object of Study}

There are 16 athletes from the women's volleyball team in Beijing Sports University, 10 of which are athletics, and 6 of which are one-level athletes, they had reduced weight for six weeks for the national youth championship meet in September 2008, the basic condition of the players are shown in Table 1. All subjects athletes have been physical examination, without abnormal phenomenon of blood pressure, heart rate etc.

\subsection{Dietary Nutrition Survey of Three Days Weighing Method}

Through three consecutive days of dietary nutrition survey tracking, and recording eating food variety and quality of athletes' three meals a day. With the letter A said the athletes' normal meal period test, with the letter B said the 
Table 1. Athletes basic table.

\begin{tabular}{|c|c|c|}
\hline Number of People & Age & Height (cm) \\
\hline \hline 16 & $20 \pm 2.83$ & $165.35 \pm 5.99$ \\
\hline Weight $(\mathrm{kg})$ & Match level & Training years \\
\hline $64.4 \pm 8.22$ & $60.5 \pm 7.59$ & $6.56 \pm 2.13$ \\
\hline
\end{tabular}

Table 2. The intake of energy materials everyday.

\begin{tabular}{|c|c|c|}
\hline Name & A & B \\
\hline \hline Total energy (kcal) & $3945.19 \pm 497.62$ & $122.31 \pm 17.97$ \\
\hline Protein(g) & $159.38 \pm 16.87$ & $145.69 \pm 24.76$ \\
\hline Fat(g) & $196.63 \pm 19.27$ & $252.13 \pm 47.17$ \\
\hline Sugar(g) & $384.19 \pm 74.77$ & \\
\hline
\end{tabular}

player control weight reduction period of testing. Specific operation is to use the range of $5 \mathrm{KG}$, and the electronic scale with the accuracy of 0.19 to weigh the quality of the food taken by athletes respectively, weighing the quality of the surplus food of the athletes again after eating respectively, and recording the data in the dietary questionnaires, the amount of extra snacks to athletes should also be tracked.

\subsection{Software Analysis and Process of Dietary Nutrition}

Using the dietary nutrition analysis software developed by the Institute of Medicine of General Administration of Sport of China to input, analyze and process the food weighing data of three days. At first input various food nutrients that athletes intake during three days of dietary weighing survey into the software in comparison to the table of food nutrients content (the content of every 100g); Then input the cooked weight of the dishes and the raw weight of vegetables, meat, spices and so on into the nutrition analysis software to calculate the proportion between, which are needed to make menu according to the recipe methods supplied by chefs; Lastly input the dietary nutrition survey data of the three days into the soft to analyze the athletes' intake situation of dietary nutrition.

\subsection{Analysis Method on Body Composition}

The body composition tests of all athletes in normal diet period and weight reduction period are respectively arranged at the end of their dietary nutrition survey of three consecutive days, with the letter A saying the normal meal period test, and the letter B saying the control weight reduction period of testing. All athletes must be kept an empty stomach, the body composition of athletes are tested by hibody 3.0 developed by the science experiment center of Beijing Sports University, the teacher of the experiment center of is responsible for the test work. The main test indicators of weight are fat weight, weight with fat, fat , the percentage of fat, intracellular fluid, extracellular fluid, total moisture, body mass index (BMI).

\section{EXPERIMENT RESULTS}

\subsection{The Intake Situation of Three Major Materials}

It can be seen from Table 2 that athletes in control weight reduction period of energy substance phase normal dietary intake and energy intake, compared to present a downward trend, especially in the total energy and carbohydrate intake value decline; Through the paired samples $\mathrm{T}$ test, energy material intake of athletes during weight reduction period has a very significant decline compare with that during normal meal period.

\subsection{The Radio of Adi And Recommended Amount of Energy Materials}

A part of the ratios in Table 3 have shown the specific value statue of ADI and recommended amount of energy materials under the current weight. In normal meal period only $18.75 \%$ of the energy of the athletes reached or exceed the recommended intake amount (by the Institute of Medicine of General Administration of Sport of China), the protein intake of $56.25 \%$ athletes reached or exceeded the recommended value, and the fat intake of all athletes were more than the recommended value by $50 \%$, and none of the carbohydrate intake had reached the recommended value; The energy intake in control weight reduction period athletes cannot meet the recommended value, only $12.5 \%$ of the athletes' energy intake is up to $80 \%$ of the recommended value, and protein intake of $12.5 \%$ of the athletes reached or exceed the recommended value, only one athlete's fat intake accounted for $97.58 \%$ of the recommended value, the fat intake for the rest of the players is more than recommended value, all the carbohydrate intake of athletes are less than $65 \%$ of the recommended value.

\subsection{Vitamin Intake Situation}

It can be seen from Table 4 that there is a downward trend the vitamin intake of athletes before and after weight reduction period, it can be seen from the average that the retinol equivalent, VB1 and VC can't get the recommended 
Table 3. The ratio of adi and recommended amount of energy materials (6 of 16).

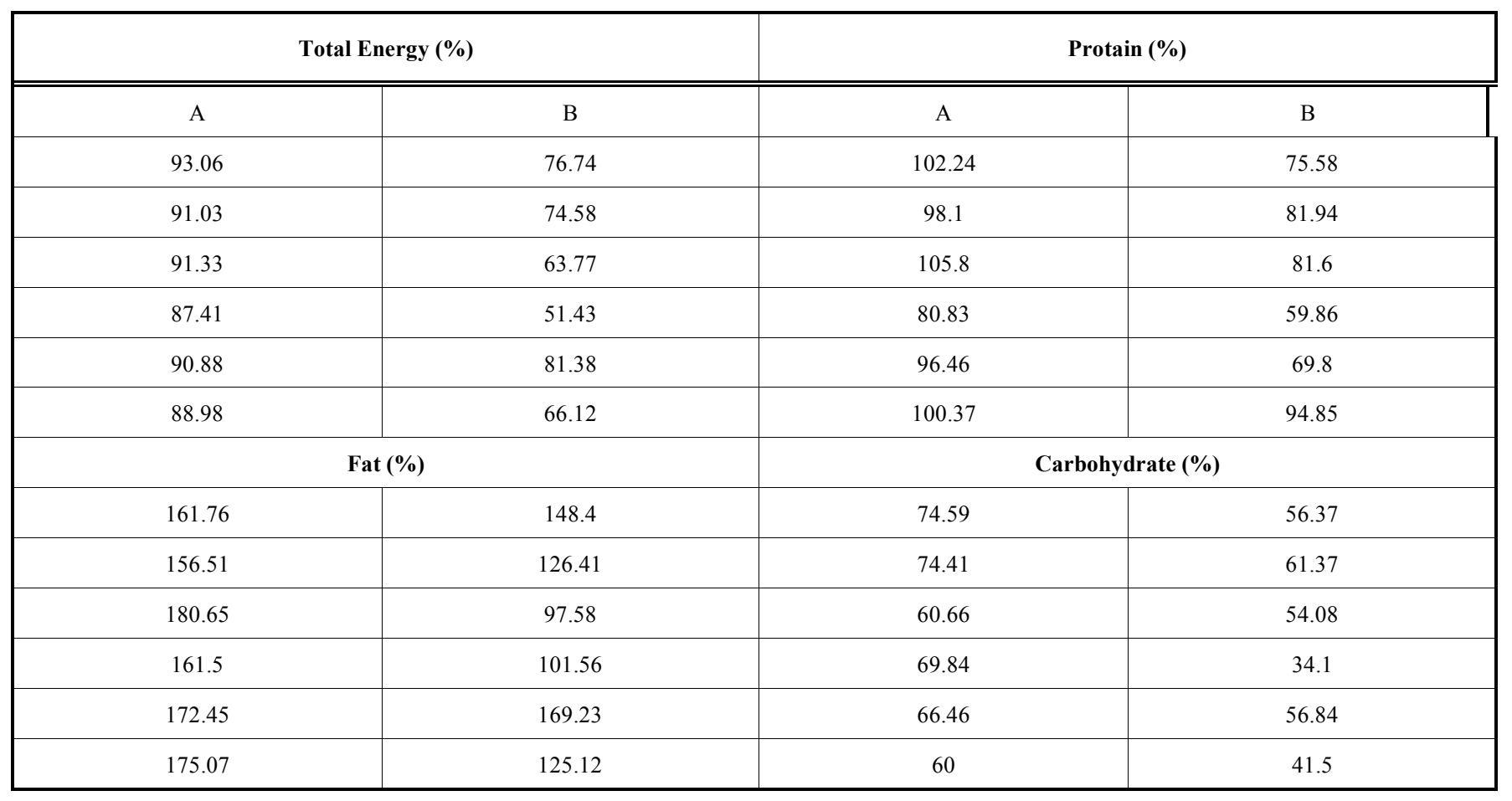

Table 4. The average situation of athletes' vitamins intake.

\begin{tabular}{|c|c|c|c|}
\hline Name & A & B & $\begin{array}{c}\text { Recommend } \\
\text { Value }\end{array}$ \\
\hline \hline $\begin{array}{c}\text { Retinol equivalent } \\
\text { (ug) }\end{array}$ & $1005.19 \pm 422.67$ & $772.80 \pm 343.21$ & 1500 \\
\hline VB1(mg) & $1.99 \pm 0.29$ & $1.60 \pm 0.39$ & 3 \\
\hline VB2(mg) & $2.00 \pm 0.18$ & $1.50 \pm 0.21$ & 140 \\
\hline VC(mg) & $128.26 \pm 22.03$ & $103.27 \pm 414.46$ & 20 \\
\hline VC(mg) & $72.67 \pm 10.40$ & $45.01 \pm 13.37$ & 8 \\
\hline Vitamins E \\
$(\mathrm{mg})$
\end{tabular}

values before and after weight reduction; On paired sample T test it was found that the intake of retinol equivalent issues during the decrease weight period has significant decline compared with normal dietary period, and the total intake of VB1, VB2, VPP and vitamin E during weight reduction period has very significant decline compared with that of the normal dietary period.

\subsection{Energy Distribution Proportion of Each Meal}

It can be seen from Table $\mathbf{5}$ that the energy intake proportion of athletes in both normal meal period and weight reduction period at breakfast can't meet the recommended value, and energy intake is more than recommended value at lunch, the energy intake of dinner basic conforms to the recommended value.

\subsection{Energy Ratio of Three Major Energy Materials}

From Table 6 it can be seen that no matter in normal meal period or weight reduction period the proportion of protein energy intake of athletes are a little more than the recommended maximum, and the energy supply proportion of fat is more than the recommended maximum power by $15 \%$, but the energy ratio of the intake carbohydrate is $20 \%$ less than the recommended minimum value.

\subsection{Variation Situation of Body Weight, Body fat, etc.} Before and After Weight Reduction Period

It can be seen from Table 7 that the average data of athletes reduced in weight reduction period than in normal meal period, of each index has very significantly decline during weight reduction stage than during normal diet. 
Table 5. Energy distribution proportion of each meal.

\begin{tabular}{|c|c|c|c|}
\hline Three meals & A & B & $\begin{array}{c}\text { Recommend } \\
\text { Value }\end{array}$ \\
\hline \hline Breakfast (\%) & $19.63 \pm 2.49$ & $23.82 \pm 4.83$ & 30 \\
\hline Lunch (\%) & $45.80 \pm 4.21$ & $45.58 \pm 5.53$ & 40 \\
\hline Dinner (\%) & $30.86 \pm 4.22$ & $29.21 \pm 3.62$ & 30 \\
\hline
\end{tabular}

Table 6. Energy ratio of energy materials.

\begin{tabular}{|c|c|c|c|}
\hline Three meals & A & B & $\begin{array}{c}\text { Recommend } \\
\text { Value }\end{array}$ \\
\hline \hline Protein (\%) & $16.21 \pm 1.37$ & $17.56 \pm 2.14$ & $12-15$ \\
\hline Fat (\%) & $45.01 \pm 2.48$ & $46.58 \pm 3.15$ & $25-30$ \\
\hline Carbohydrate (\%) & $38.63 \pm 3.05$ & $35.81 \pm 2.89$ & $55-65$ \\
\hline
\end{tabular}

Table 7. Variation situation of body weight, body fat before and after weight reduction period.

\begin{tabular}{|c|c|c|}
\hline Name & A & B \\
\hline \hline Weight(kg) & $64.4 \pm 8.22$ & $52.24 \pm 7.9$ \\
\hline FFM(kg) & $5.35 \pm 6.41$ & $10.24 \pm 2.03$ \\
\hline Fat $(\mathrm{kg})$ & $10.9 \pm 2.17$ & $16.38 \pm 1.89$ \\
\hline Fat percentage $(\mathrm{kg})$ & $128.26 \pm 22.03$ & $22.87 \pm 1.82$ \\
\hline BMI $(\mathrm{kg} / \mathrm{m} 3)$ & $23.67 \pm 1.94$ & \\
\hline
\end{tabular}

\subsection{The Index Changes in Blood Lipids}

It can be seen from Table $\mathbf{8}$ that except the average value of free fatty acids, high density lipoprotein ( HDL-C) and apolipoprotein A of athletes during the weight reduction period rose, the value of the rest blood fat index decreased. Free fatty acids significantly increased after weight reduction period, the high density lipoprotein ( HDL-C ) and apolipoprotein A increased very significantly, and triglyceride significantly decrease remarkable, apolipoprotein B decreased very significantly; The values of low density lipoprotein cholesterol (LDL-C) and very low density lipoprotein cholesterol (VLDL-C), total cholesterol only performed decline.

\section{ANALYSE AND DISCUSS}

\subsection{Analysis and Discussion of Three Days Dietary Nu- trition Survey}

Protein, fat, carbohydrates are the main material to provide energy for the body, the intake of them is too much or too little in the dietary can affect the body's physiological function. Only athletes accomplish rational diet, can the intake of three major power material can be guaranteed balanced and reasonable, so as to provide material guarantee for sports training. Protein is the main form of life; it is also the life important active material that constitutes human body, the content of protein in human body is about $16 \%$ of the weight [1-2]. Protein is the main component of cell, accounting for over $80 \%$ of the solids in the cell. Muscle, blood, keys, bones and cartilage etc. are all composed of protein. Metabolism and the damaged tissues in the body also need to the repair of protein. Therefore, the proteins maintain the growth, update, and repair of tissues. Plasma protein can maintain the osmotic pressure of the body, protein is also the components of buffer system in the body, to maintain the acid-base balance, some amino acids in proteins are the materials for the synthesis of ethyl phthalein choline, therefore it also has relationship with the conduction and excitement of nerve tissue. Studies have shown that the protein can increase the excitability of the central nervous system, and individual amino acids such as methionine and lysine contribute to the establishment of conditioned reflex. In addition, protein can strengthen the body immunity, and experiments show that when protein is malnourished, the body's immune function decrease, leukopenia happens, and the phagocytosis of white blood cells and reticulocyte endothelial cell decline $[3,4]$.

Fat is one of three major supply of nutrients, its heat yield is very high, $1 \mathrm{~g}$ fat oxidation to produce $37.65 \mathrm{~kJ}$ energy in the body. But compared with carbohydrate, fat has the characteristic of light weight, high energy density, high 
Table 8. The index changes in blood lipids.

\begin{tabular}{|c|c|c|c|}
\hline Name & A & B & $\begin{array}{c}\text { Recommend } \\
\text { Value }\end{array}$ \\
\hline \hline $\begin{array}{c}\text { Free fat acid (mmol/L) } \\
\text { HDL-C } \\
\text { (mmol/L) }\end{array}$ & $0.34 \pm 0.14$ & $0.60 \pm 0.33$ & $1.56 \pm 0.28-0.77$ \\
\hline $\begin{array}{c}\text { LDL-C } \\
(\mathrm{mmol} / \mathrm{L})\end{array}$ & $1.40 \pm 0.26$ & $2.61 \pm 0.38$ & $1.09-2.06$ \\
\hline $\begin{array}{c}\text { VLDL-C } \\
(\mathrm{mmol} / \mathrm{L})\end{array}$ & $2.77 \pm 0.51$ & $0.37 \pm 0.22$ & $0.2-0.9$ \\
\hline Apolipoprotein A (g/l) & $0.45 \pm 0.16$ & $1.36 \pm 0.14$ & $1.22-1.61$ \\
\hline Apolipoprotein B $(\mathrm{g} / \mathrm{l})$ & $1.24 \pm 0.11$ & $0.59 \pm 0.07$ & $0.69-1.05$ \\
\hline $\begin{array}{c}\mathrm{TG} \\
(\mathrm{mmol} / \mathrm{L})\end{array}$ & $0.72 \pm 0.11$ & $0.81 \pm 0.25$ & $0.6-1.7$ \\
\hline $\begin{array}{c}\mathrm{TC} \\
(\mathrm{mmol} / \mathrm{L})\end{array}$ & $1.27 \pm 0.61$ & $4.46 \pm 0.48$ & $3.1-5.7$ \\
\hline
\end{tabular}

calorific value, etc. Fat in dietary can supply unsaturated fatty acids that body needs, and unsaturated fatty acids are the important components for the cell membrane, enzyme, mitochondria lipoprotein, and have a certain auxo-action to reproduction and sexual maturity [5]. But excessive intake of essential fatty acids may also have some side effects, such as weakening the antioxidant capacity of the body, producing a large number of peroxides, inspiring free radical formation and causing damage to the body. The body fat can also promote the absorption and utilization of fat-soluble vitamins A, $\mathrm{D}, \mathrm{E}, \mathrm{K}$, and can also increase the delicious, give full feel to body. Body fat can also form a fat pad bag around the surrounding organs, to protect the organs and nerve from trauma. Sterols is the raw material of cholesterol, vitamin D, sex hormones and adrenal cortical hormone, cholesterol is the transportation of unsaturated fatty acid, so the metabolic disorder also has close relationship with atherosclerosis $[6,7]$.

Carbohydrates (sugar) is one of the most important energy sources materials in the body, the athletes taking balanced mixture dietary carbohydrates supply according to the calculation of calorific value is about $60 \%$ of the total energy. Western countries generally recommend the intake of sugar should be at least $50 \%-50 \%$ of total energy (the national health and medical research council), some authoritative advice to increase the intake of sugar to $65 \%$ of the total energy for long time exercise, and the carbohydrate supply of great intensity endurance training athletes should be $60 \%$ $70 \%$ of the total energy, the supply of moderate intensity exercise should be $50 \%-60 \%$ [8].

The survey found that the total energy and the intake of energy material of the athletes during the weight reduction period showed very significant decline compared with normal dietary intake and period, especially the intake value of the total energy and carbohydrate have the maximum decline; Compared with normal meal period, the energy intake of the athletes from women's volleyball team in Beijing Sports University are $1134.75 \mathrm{kcal}$ less a day on average during weight reducing period, which shows that the athletes pay full attention to reduce dietary total energy intake in order to lose weight control and Reducing dietary energy intake is the main method to reduce weight slowly. But athletes tend to lack scientific and reasonable dietary guidance when reducing weight, as a result, the proportions of protein, fat, and carbohydrate intake are not reasonable.

This study found that the energy intake of women's volleyball athletes of three meals a day also does not conform to the recommended value. The proportion energy intake of breakfast is obvious insufficient which shows that athletes do not have breakfast or just have a precise breakfast, but they also have the training task in the morning, poor breakfast will not be able to fully guarantee the effect of training. Fat intake should be appropriately reduced at dinner, at the same time, containing carbohydrates drinks and protein should be appropriately supplied, it helps athletes with their recovery after one day training. So three meals a day should be paid great attention by athletes, and athletes should have good breakfast, satisfied lunch, and little dinner, balance energy intake of meals well, store reasonable energy for one day sports training.

And in this investigation the energy proportion of the three major energy material that athletes intake is unreasonable, the energy proportion of fat is higher than the recommended value, this is mainly associated with the excessive intake of fat, and it is also associated with too little carbohydrate intake, and high energy proportion of protein is mainly because of the too little energy proportion of carbohydrate. So athletes should reduce fat intake, increase the carbs and guarantee the balance of energy proportion of energy material, so as to achieve good effect in sports training.

\subsection{Analysis and Discussion on the Intake Status of Vi- tamins}

Vitamins are species of essential organic compounds that maintain normal physiology functions and healthy of human 
body, a little amount of them can fulfill the need of normal physiology functions but lack of them cannot be accepted because obstruct of physiology functions and disease will appear as the lack of vitamins. This diet nutrient survey discovers that, there are differences between each person as to the intake of vitamins and the diet nutrient survey questionnaires show that it is mainly associated with individual eating habit and preferences of athletes. But as to all of the athletes, except the intake of VPP and vitamin E can attach the recommend value, there are lack phenomenon of the intake of retinol vitamin B1, B2, C which shows that the intake of vitamins should be paid attention to by both coaches and athletes。Epidemiological studies prove that retinol lack causes reduce of organism cellular immunity function [1]. $\mathrm{VC}$ has the properties of antioxidant, and can promote the absorption of iron, strengthen human body immunity, mild fatigue is the early performance of lack of $\mathrm{VC}$, when it is serious symptoms of scurvy will appear [4].

This survey found that some athletes are easy to catch cold or be inattention during sports training in weight reduction period, this may be associated with the lack vitamin $\mathrm{C}$ of some athletes, and lack of $\mathrm{VC}$ in diet is the main factor that causes a deficiency of the vitamin. And the lack of other vitamins should also be brought to forefront; there should be more supplements for the lack vitamins especially during weight reduction period. The coaches and athletes should pay full attention to the importance of vitamins to a healthy body; they must ensure the enough intakes of athletes' vitamins.

\subsection{Analysis and Discussion on the Change of Blood Bio- chemistry Indexes}

There are $67 \%-80 \%$ of cholesterols in LDL in blood serum, so the rise of blood serum TC will result in the rise of LDL-C necessarily. The rise of LDL is the main lipid dangerous element of, so LDL-C test can be regarded as the risk access of atherosclerotic diseases such as coronary heart disease. TG is the main basis of, the differences of TG level in and between individuals are both bigger than that of TC, which will lead to the disadvantages of the decrease of HDL$\mathrm{C}$ and the rise of LDL-C, and give rise to arteriosclerosis hardening of the arteries indirectly. HDL is a kind of lipoprotein which has the highest medium density and the smallest particles; it is the foundation of blood lipid metabolism, specifically combining with extra blood lipids in the body, transmitting out to vitro, removing blood trash. And due to the small size, it can penetrate artery intima to remove and carry the cholesterol depositing inside out of vessel wall, repair vascular endothelial damage cells. HDL has the function of restoring the elasticity of blood vessel, removing excess fat and cleaning the role of blood vessels, and it is known as the real intravascular lipid scavenger; HDL can fade atherosclerosis plaques, resume the function of endothelial cells, vascular endothelial injury and prevent LDL oxidation, reduce or prevent LDL from damaging endothelial cell, etc., so it is called resistance factor of hardening of the arteries [2].

The biochemistry monitor on various blood fat indexes in this research is to understand the influence of diet change on blood fat supersession before and after weight reduction period. It mainly analyzes and explores the influence of diet change on HDL-C, meanwhile avoids the confusion of blood fat supersession arouse by controlling weight, which can affect body healthy level of athletes. Research shows that except the average value of free fatty acids, apolipoprotein A and HDL-C rise during weight reduction period, other various index of blood fat all descend.

The change of HDL-C and apolipoprotein A present the consistency in the research, the rising of their values can increase the ability of reversal transport cholesterol, so the change of these two indexes are changing to the advantage directions on blood fat supersession, that is mainly associate with the influence of the total energy intake decreasing of diet nutrient and sports training, and it is especially associate with the increase of appropriate aerobic exercises during weight reduction period.

\section{CONCLUSION}

Among the dietary intake of athletes in women's volleyballteam in Beijing Sports University during weight direction period, the intake of total energy is not enough, the intake of fat is out of limits, and the intake of carbohydrates, VB1, VB2 and VC are decline. As to the energy distribution proportion of daily meals, the proportion of breakfast can't meet the recommended value, the proportion of lunch was above the recommended value, and the proportion of dinner was in line with recommended value. The energy percentage of three major dietary energy material did not conform to the recommended values, the energy percentage of fat was too high, and the energy percentage of carbohydrate was insufficient. Diet control combined with exercise training can effectively reduce body weight, body fat, body fat percentage and BMI, it also causes the decline of fat free mass and the water content in the body. Appropriate diet control can cause the increasing of high density lipoprotein and apolipoprotein A and free fatty acids, and at the same time cause the decreasing of apolipoprotein B and triglycerides, which is advantage to blood lipid metabolism. The levels of serum testosterone and serum glucose of athletes dropped during weight reduction period.

So women's volleyball athletes should increase the intake of carbohydrates and vitamins, reduce the intake dietary fat, and reduce oil during the process of cooking. Athletes should form the habit of having good daily meals, achieve as , to have a good breakfast, to have a satisfied lunch, and have a less eat dinner. Lighter food should better be taken in dinner and fat intake reduce should be reduced. Athletes should appropriately increase the intake of protein during weight reduction period, to maintain lean body mass. Athletes should increase water intake during weight reduction period, and increase the intake of sugar water and complex sports drinks appropriately. Athletes should better have low fat diet and take aerobic exercises during weight reduction period.

\section{CONFLICT OF INTEREST}

The author confirms that this article content has no conflict of interest. 


\section{ACKNOWLEDGEMENTS}

This work is supported by the Key Project of Guangxi Social Sciences, China (No. gxsk201424), the Education Science fund of the Education Department of Guangxi, China (No.2014JGA268), and Guangxi Office for Education Sciences Planning, China (No.2013C108).

\section{REFERENCES}

[1] J. Cao, Y. Tain, etc. "Exercise with iron metabolism," Journal of Beijing Sports University May Evening 00, vol. 26, no. 3, pp. 331335.

[2] J. Chen, "Sports nutrition," Beijing: Beijing medical university press, vol. 8, no. 4, 2002.
[3] X. Dong, H. Guo, and Y. Mo, "To drop weight athletes to the study of evaluation function," Journal of Beijing sports university, vol. 30, no. 9, pp. 1299-230[2] 2007.

[4] J.A. Nogueira, and T.H. Da Costa, "Nutrient intake and eating habits of triathletes on a Brazilian diet Diet," Int J Sport Nutr Exere Metab, vol. 14, no. 6, pp. 684 697, 2004.

[5] F. Magkos, and M. Ynnakoulia, "Methodology of dietary assessment in athletes: concepts and pitfalls," Curr Opin Clin Nutr Metab Care, vol. 6, no. 5, pp. 539-549, 2003.

[6] A. Qu, and J. Zhao, "1208 cases of healthy blood lipid levels analysis," Practical medical journal, vol. 14, no. 3, pp. 351-353, 2007.

[7] A. Zhang, "Medical nutrition," Beijing: people's medical publishing house, pp. 25-46, 1998.

[8] Z. Zhao, L. Xue, and Y. Hu, "Function evaluation, etc. The modern sports medicine, medical supervision and athletic injury protection, clinical diagnosis and treatment, health and nutrition practice encyclopedia (3) [MI. Huhhot: the distance press, 1492-1551, 2005.

Received: June 10, 2015

Revised: July 29, 2015

Accepted: August 15, 2015

(C) Xiaolin Wang; Licensee Bentham Open.

This is an open access article licensed under the terms of the (https://creativecommons.org/licenses/by/4.0/legalcode), which permits unrestricted, noncommercial use, distribution and reproduction in any medium, provided the work is properly cited. 